\title{
Un récupérateur de chute incorporé à la galerie de fuite d'une usine souterraine
}

\section{A head recovery structure incorporated in the tailrace tunnel of an underground power house}

\section{P. STMON}

INGÉNIEUR EN CHEF A ÉLECTRIGITÉ DE FRANCF CHEF DE LA MISSION D'ÉTUDES DU KONKOURQ́
PAR

ET

\author{
J. VALLÉE
}

INGÉNIEUR A ÉLECTRICITÉ DE FRANCE INSTECTION GÉNÉRALE POUR L'UNION FRANGAISE ET L'ÉTRANGER

\begin{abstract}
Le projet d'aménagement de Souapiti (Guinée), auec son barrage en terre de $120 \mathrm{~m}$ de hauteur sur le Konkouré, comporte une usine souterraine produisant plus de 3 milliards de $k$ Wh. La res. titution des eaux all Konkouré s'effectue à travers une galerie de fuite de $6200 \mathrm{~m}$ de longneur et de $114 \mathrm{~m}^{2}$ de section. La galerie a été calée de manière à faciliter au maximum l'exécution des travaux. Il s'ensuivait une perte de chute dont on peut finalement récupérer une grande partie grâce au dispositif décrit dans cet article. La galerie à deux pentes : $1.95 \%$ sur le troncon amont, de $4800 \mathrm{~m}$ de longueur et $0,5 \%$ pour le tronçon aval, de $1400 \mathrm{~m}$ de longueur. Les ouvrages incorporés sont conçus comme suit:

- à l'extrémité aval, l'onvrage récupérateur, en forme de siphon, est destiné à créer un entrainement d'air permanent; la galerie de fuite fonctionnant $\dot{a}$ écoulement libre, ce débit d'air tend à créer dans la galerie une atmosphère déprimée; la dépression constitue la chute récupérée;

- a l'amont de la galerie, un organe hydraulique permet de contróler la dépression en laissant rentrer de l'air dès que cette dépression dépasse la valeur choisie.

L'air évacué par l'ouvrage aval provient du dégazage de l'eau soumise à la dépression, des fuites éventuelles dans le rocher et de l'appoint d'air laché par le régleur de dépression.

Les essais ont montré que l'on pouvait escompter en toutes circonstances une dépression de $4,50 \mathrm{~m}$. Cette récupération de $4,50 \mathrm{~m}$ représente un supplément de production de 100 millions de liwh.
\end{abstract}

Le projet d'aménagement du Konkouré en Guinée comporte, outre le barrage de Souapiti, de $120 \mathrm{~m}$ de hauteur, permettant la régularisation annuelle et interannuelle, et l'usine souterraine capable d'une production de plus de 3 milliards

\begin{abstract}
The Souapiti (Guinée) development with its 120 metre high earth dam on the Konkouré river includes an underground power station with an output of more than $3,000,000,000 \mathrm{kWh}$. The tailrace consists of a 6,200 metre long tunnel with a cross-sectional area of 114 square metres. The setting adopted for the tunnel was such as to facilitate construction work. This resulted in a loss of head which is recoverable by means of the structure described in this article.

The tunnel has two gradients: $1.95 \%$ over the 4,800 metre long upstream section, and $0.5 \%$ over the 1,400 metre long dounstresm section. The following structures are incorporated in the tunnel:

- At the downstream end; the head recovery structure consisting of a siphon designed to entrain air continuously. Since the tunnel is of the gravity type, air entrainment through the siphon sets up a partial vacuum in the tunnel thus causing a recovery of head;

- At the upstream end; a hydraulic control device which allows air to enter the tunnel when the vacuum exceeds a preselected value. The air extracted by the downstream structure consists of formerly dissolved air freed from the water flowing under partial vacuum, of any air that has leaked through the rock and of air released into the tunnel by the vacuum controlling device.

Tests have shown that a vacuum of 4.5 metres is available under all conditions. This 4.5 metre head represents an extra output of $100,000,000 \mathrm{kWh}$.
\end{abstract}

de $\mathrm{kWh} / \mathrm{an}$, une galerie de fuite de grande longueur permettant l'utilisation de près de $40 \mathrm{~m}$ de chute naturelle.

Cette galerie, par sa section de $114 \mathrm{~m}^{2}$ et sa longueur de $6200 \mathrm{~m}$, posait des problemes de 
fonctionnement et d'exécution dont certains devaient trouver une solution par la conception même de l'ouvrage, en particulier en ce qui concerne le calage et la pente à adopter pour cet ouvrage."

Pour utiliser intégralement la chute disponible, ii aurail fallu prévoir une galerie fonctionnant complètement en charge et par conséquent débouchant en dessous du niveau naturel des eaux au point de restitution.

En raison de la grande hauteur de la galerie, on devait par conséquent abaisser d'autant, soit de $11 \mathrm{~m}$, l'ensemble de celle-ci.

Cette solution posait des problèmes d'exécution, telle l'évacuation de plusieur's centaines de milliers de mètres cubes de déblais, et surtout un problème de sécurité du personnel et de poursuite des travaux en cas de venues d'eau importantes et non prévisibles.

Pour éviter toutes ces difficultés et leur répercussion économique sur le projet, la galerie doil présenter une pente descendante depuis l'usine et déboucher au moins au-dessus du niveau des basses eaux du Konkouré.

Ces conditions élanl imposées, il restait à choisir entre les deux possibilités suivantes et à ćvaluer dans chaque cas la perte de chute qui en résultait :

-- adapter pente et section au débit, de manière que la galerie se mette en charge d'ellemême par l'amont,

-_ donner à la galerie une pente telle que l'écoulement ait lieu à surface libre.

La première solution, dite à écoulement en charge, a été abandonnée, car elle présente un inconvénient grave; le mode d'écoulement est mixte en réalité : en charge à l'amont, libre vers l'aval. Le décollement qui se produit au passage de l'écoulement en charge à l'écoulement libre risquait d'être gênant. Le point de décollement pourrait en effet se déplacer, d'autant plu's que l'aération se ferait par l'aval, et l'écoulement présenterait sans doute une instabilité à laquelle il serait difficile de remédier pour tous les débits.

La solution à écoulement libre par contre, était préférable du point de vue de l'exploitation; encore fallait-il ajuster la pente de manière à limiter au minimum la chute non récupérable.

Pour établir sur toute la longueur de la galerie un écoulement dont le régime uniforme à l'amont donne la débitance maximum pour le débit équipé, $340 \mathrm{~m}^{3} / \mathrm{s}$, la pente était uniformément de $1,95 \%$, compte tenu de la section et de la rugosité admise pour les parois. Le coefficient de Strickler adopté est de 32 .

Si l'on ne dispose à l'aval de la galerie d'aucun seuil, le débit s'écoule à l'extrémité aval sous la hauteur critique, moitié environ de la profondeur normale du régime uniforme. Il en résulte, dans la partie aval de la galerie, un abaissement du plan d'eau qui n'est autre que la courbe de remous raccordant le niveau de l'extrémité aval au niveau de l'écoulement uniforme vers l'amont (fig. 1).

Il est possible de tirer parti de cette remarque pour gagner déjà 2 mètres de chute environ. Il suffit de donner une pente faible, c'està-dire la pente minimum de $0,5 \%$, dans la partie aval de la galerie. Cette pente faible est conservée jusqu'à ce que la ligne de remous atteigne la profondeur normale correspondant à l'écoulement uniforme amont avec la pente de $1,95 \%$.

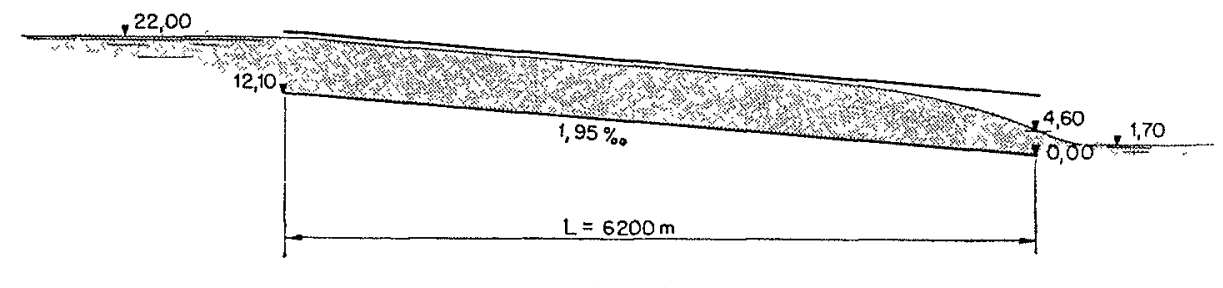

FIG. $1 a$

Galerie à pente unique. Ecoulement libre.

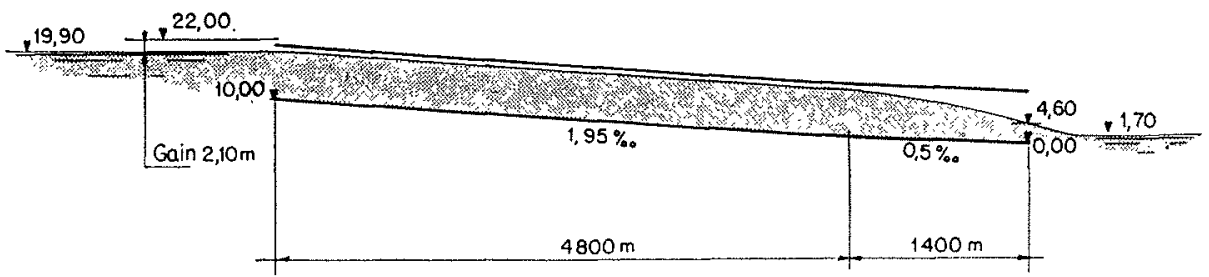

Fic. $1 b$

Galerie à pente mixte. Ecoulement libre. 
On arrive ainsi à une galerie à deux pentes : $1,95 \%$ pour le tronçon amont, de $4800 \mathrm{~m}$ de longueur, et $0,5 \%$ pour le tronçon aval, de $1400 \mathrm{~m}$ de longueur.

Cette solution, déjà satisfaisante, ne permettrait pas cependant, à elle seule, d'utiliser intégralement la chute disponible. En effet, la solution à pente unique donnait pratiquement une perte de chute de $8 \mathrm{~m}$ environ. La pente mixte assurant le gain de $2 \mathrm{~m}$, il restait donc près de (6 m non utilisés.

\section{Récupérateur de chute}

Pour ne pas abandonner ces quelques mètres de chute, les ingénieurs d'Electricité de France, auteurs du projet, eurent alors l'idée de prolonger l'extrémité aval de la galerie par un ouvrage en béton débouchant au-dessous du niveau des eaux du Konkouré, ouvrage rappelant par sa forme un siphon disposé à l'envers.

Cette conception originale a fait l'objet d'études sur modèle qui furent confiées à la SOGREAH à Grenoble.

Ces études et les mises au point sur modèle ont conduit au système qui comporte finalement :

- A l'extrémité aval, l'ouvrage récupérateur schématisé par la figure 2 .

La mise en vitesse de l'eau obtenue à travers cet ouvrage permet l'entraînement d'un certain débit d'air, créant ainsi dans la galerie une atmosphère déprimée au-dessus du plan d'eau. Il s'ensuit un fonctionnement à écoulement libre «en dépression » et la va-

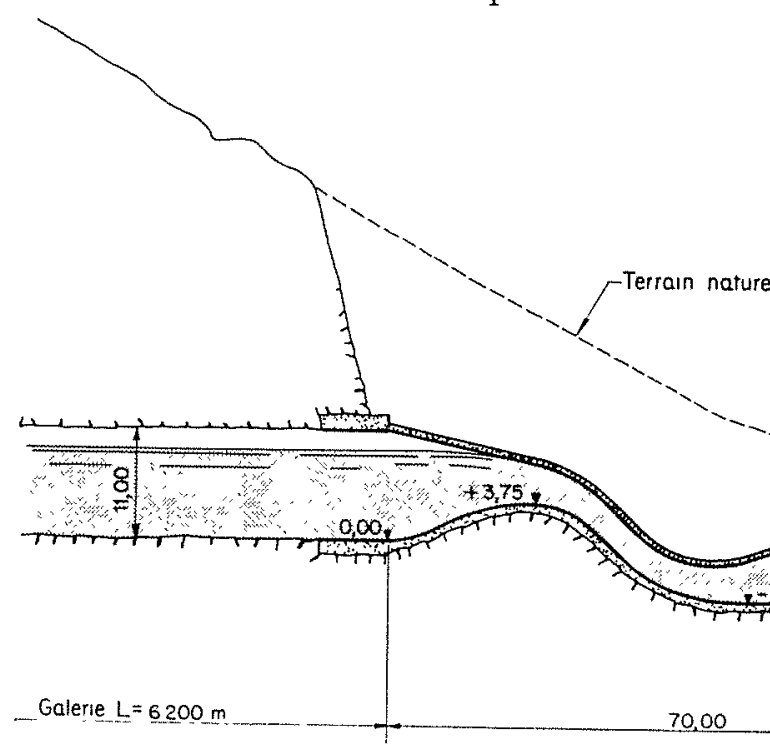

Ieur de cette dépression constitue la chute récupérée.

L'ouvrage comporte d'abord un seuil, qui a pour but essentiel de maintenir dans la galerie un niveau suffisant pour limiter à une valeur acceptable la vitesse de l'eau dans la partie aval de la galerie de fuite. Au-delà du seuil, le radier plonge vers l'aval et la section offerte au passage de l'eau se rétrécit de manière à assurer la mise en vitesse capable de provoquer l'entraînement d'air et d'établir ainsi la dépression recherchée.

La partie supérieure de cet ouvrage est constituée par une dalle en béton soutenue par trois cloisons et raccordée à la calotte de la galerie par une forme simple.

- A lamont de la galerie de fuite, un dispositif hydraulique permet de contrôler la dépression en laissant rentrer de l'air dès que cette dépression dépasse la valeur choisie.

Un tel dispositif n'apparaît pas indispensable au fonctionnement du récupérateur de chute, mais en raison de l'importance des ouvrages, il a été jugé utile de le prévoir.

Ce régleur hydraulique est placé sur le reniflard reliant la galerie à la cheminée d'équilibre (fig. 3).

La cheminée d'équilibre et le reniflard ont été prévus pour assurer le fonctionnement normal de la galerie lor's d'une deuxième étape qui verrait la mise en service d'un barrage, projeté à l'aval, dont la retenue mettrait la galerie de fuite en contrepression. Le reniflard servira donc, dès la première étape, à écoulement libre, au contrôle de l'aération.

Fig. 2

Coupe de principe du récupérateur. 


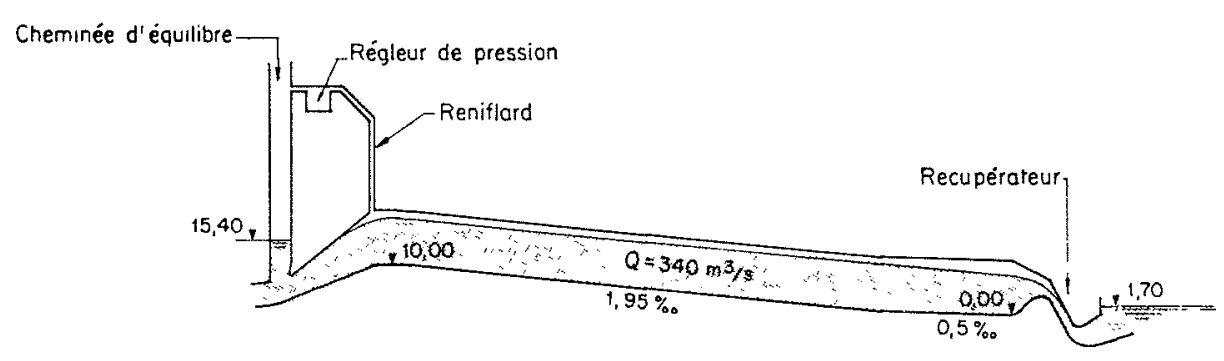

Fig. 3

Schéma d'ensemble de la galerie de fuite.

Le régleur hydraulique, comme le montre la figure 4 , se compose d'une chambre séparée en deux compartiments par une cloison centrale. Les deux compartiments communiquent dans leur partie basse, car la cloison n'est pas descendue jusqu'au radier.

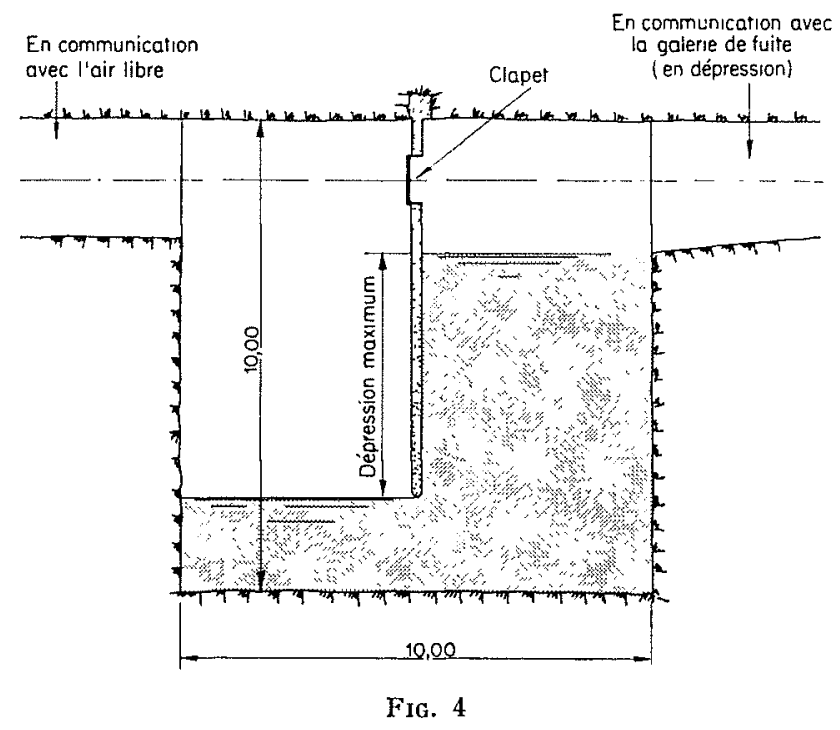

Régleur hydraulique de pression.

La différence de pression entre l'amont et l'aval de la cloison maintient une différence de niveau égale à la dépression dans la galerie. Au-delà d'une certaine valeur, déterminée par la hauteur de la cloison, le niveau d'eau amont découvre le bas de cette dernière pour laisser passer de l'air, ce qui réduit la dépression et, en fait, la limite à la valeur choisie.

La dépression réalisée dans la galerie est d'ailleurs représentée par la différence de niveau existant entre le plan d'eau de la cheminée d'équilibre qui est à la pression atmosphérique et le plan d'eau à l'entrée de la galerie, au droit du reniflard.

L'ensemble de la galerie et de l'ouvrage aval constitue en quelque sorte un siphon débitant de l'eau et de l'air.

L'air ainsi évacué par l'ouvrage aval provient :

- du dégazage de l'eau soumise à la dépression;

- des fuites éventuelles dans le rocher, car la galerie en principe n'est pas revêtue;

- de l'appoint d'air lâché par le régleur de dépression.

La quantité d'air libéré par l'eau soumise à une dépression étant fonction de cette dépression, un point d'équilibre s'établirait même sans l'intervention du régleur.

Les essais ont montré que l'on pouvait considérer qu'une dépression de $4,50 \mathrm{~m}$ serait obtenue en toutes circonstances.

Cette récupération de $4,50 \mathrm{~m}$ supplémentaires, qui garantit pratiquement l'utilisation complète de la chute disponible, représente un appoint de production de 100 millions de $\mathrm{kWh}$ par année. Il est évident qu'il s'agit là d'une énergie marginale à extrêmement bon marché. 